\title{
Article \\ Coupling of External Electric Circuits with Computational Domains
}

\author{
Riccardo Scorretti
}

Citation: Scorretti, R. Coupling of External Electric Circuits with Computational Domains. J 2021, 4 , 865-880. https://doi.org/10.3390/ j4040059

Academic Editor: George

Kosmadakis

Received: 27 October 2021

Accepted: 24 November 2021

Published: 14 December 2021

Publisher's Note: MDPI stays neutral with regard to jurisdictional claims in published maps and institutional affiliations.

Copyright: (C) 2021 by the author. Licensee MDPI, Basel, Switzerland. This article is an open access article distributed under the terms and conditions of the Creative Commons Attribution (CC BY) license (https:// creativecommons.org/licenses/by/ $4.0 /)$.
Univ Lyon, Université Claude Bernard Lyon 1, INSA Lyon, ECLyon, CNRS, Ampère, F-69100 Villeurbanne, France; riccardo.scorretti@univ-lyon1.fr

\begin{abstract}
Coupling of electrical circuits with 2D and 3D computational domains is very important for practical applications. To this aim, the notions of "electrical current" and "voltage" must be defined precisely and linked with local quantities (i.e., fields and potentials) in the computational domain. Apart from the static case, the definition of voltage is more complex than it may appear at a first glance, and it is usually tainted by unspoken and/or not justified assumptions. The purpose of this work is twofold: on one hand, to shed light on the definition and on the physical meaning of voltage in the case of time varying quasi-static fields and, on the other hand, to show how to establish coupling equations between lumped parameters circuit model and 2D/3D computational domains. It is demonstrated that a precise physical significance can be given to the voltage in terms of power balance only (the notion of potential is unnecessary). A couple of original operators which allow to express voltages and currents are introduced. Based on a critical analysis of the research literature, it is shown that existing coupling formulas can all be rewritten as particular cases of these two operators. The developed analysis is independent from any computational method and can be used to devise new coupling formulas.
\end{abstract}

Keywords: finite element analysis; electrical circuits; eddy currents

\section{Introduction}

Coupling of external electrical circuits with Finite Element (FE) computational domains is very important for practical applications, and it has been investigated for a very long time (see [1] for a historical perspective in 1993). Coupling external circuits with 2D/3D computational domain is necessary to model electrotechnical appliances such as simple coils [2,3], non linear inductors [4], contactors [5,6], power transformers $[7,8]$, electrical motors [9-11] and many others [12-15]. Maxwell's equations are solved in the computational domain by using various formulations, the unknown of which are local quantities such as potentials, or (less frequently) the magnetic fields directly [16-19]. Currents and voltages are global quantities that are applied to the computational domain through "ports", that is, interfaces between internal regions, or surfaces on the external boundary of the domain.

To this aim, the notions of "current" and "voltage" must be precisely defined and linked with the electric field $\mathbf{e}$ and current density $\mathbf{j}$ in the computational domain. The definition of the electrical current $I$ which flows across a given surface $\Sigma$ is easily expressed and it depends uniquely on the current density:

$$
I=-\int_{\Sigma} \mathbf{n} \cdot \mathbf{j}
$$

where $\mathbf{n}$ is the unit normal vector, with outward orientation in respect of the computational domain. Conversely, the understanding of the physical significance of voltage is not as trivial as it may appear [20], and it is worth to be clarified. In the case of static fields 
(electrostatics, continuous currents), the electric field is conservative and the voltage $U$ between two equipotential surfaces $\Sigma_{a}$ and $\Sigma_{b}$ can be uniquely defined as:

$$
U=\int_{\gamma} \mathbf{e} \cdot \mathbf{d} \mathbf{l}=v_{a}-v_{b}
$$

where $\gamma$ is any path which goes from $\Sigma_{a}$ to $\Sigma_{b}$ and $v$ is an electric scalar potential such that $\mathbf{e}=-\operatorname{grad} v$. However, this definition of voltage cannot be truly satisfying in that it does not apply and cannot be easily extended to the case of time varying fields. In fact, in this case, the electric field is no more conservative and thus the choice of the path $\gamma$ matters.

In the case of time varying fields, one may define the voltage as the circulation of the "electrostatic component" (- grad $v)$ of the electric field:

$$
\mathbf{e}=-\partial_{t} \mathbf{a}-\operatorname{grad} v
$$

However, in the case of bounded domains, there is no such thing as the electrostatic component: at most, one may speak of an electrostatic component. In fact, Helmholtz's theorem [21] ensures that such a decomposition (3) exists, but in the case of bounded domains it is not unique [22] unless appropriate additional boundary conditions are imposed. Even assuming that such a definition is well posed from the mathematical point of view - that is, if one assumes that the value of the voltage hereby defined is independent on the gauge of $(\mathbf{a}, v)$, which is indeed the case-the question of the physical significance arises.

One observes that the definition (1) is not tainted by any of these issues: the current $I$ is defined based on the current density only, and the definition is unambiguous in the case of time varying fields, and whatever the surface $\Sigma$.

The question of the well-posedness of the definition of voltage, in the case of time varying fields, is seldom addressed in undergraduate courses in physics and electrical engineering (because in practice "things work") but also in most of the scientific literature. In the author's opinion, any effort to shed light on this topic is worth to be explored.

The case of stranded coils $[2,3,7,8]$ is considerably simpler. In fact, isopotential surfaces $\Sigma_{a}$ and $\Sigma_{b}$ reduce to points, so that in practice the integration path $\gamma$ is unique, and it is imposed by the geometry of the electrical wires. In this case, the induced electromotive force can be expressed through the time derivative of the magnetic flux.

The case of solid conductors is more complex. In some cases [10], the voltage between two "isopotential" surfaces is mathematically defined as the difference of an electric scalar potential $v$, but the question of its physical significance is eluded. As already observed, this scalar potential is not unique and it is not clear at all why the voltage has the same value whatever the gauge of $(\mathbf{a}, v)$. The very same notion of "isopotential" surfaces is disturbing, because in the case of bounded domains it depends on a particular choice of the gauge, whereas the voltage ought to depend uniquely on the electric field.

The same considerations apply to $[4,11,14]$, where a $2 \mathrm{D}$ modelling is taken on. In this case, Coulomb's gauge div $\mathbf{a}=0$ is arbitrarily fixed by imposing that the vector potential a is perpendicular to the $x y$ plane, and thus a voltage between the front and the rear part of the domain can be uniquely defined as:

$$
U=\ell(-\mathbf{n} \cdot \operatorname{grad} v)
$$

where $\ell$ is the depth of the domain and $\mathbf{n}$ is the unit vector perpendicular to the plane.

In [6,23], the following relationship is derived for a two-ports conductor in the electrokinetic case (also known as continuous current):

$$
U=-\int_{\Omega} \mathbf{j}_{0} \cdot \operatorname{grad} v
$$


where $\mathbf{j}_{0}$ is any current density corresponding to a net current of $1 \mathrm{~A}$. This equation is then generalised in various "flavours" $[6,13,23]$, to the case of time varying fields, by rewriting $\operatorname{grad} v=-\partial_{t} \mathbf{a}-\mathbf{e}:$

$$
\begin{aligned}
U & =\int_{\Omega_{c}} \mathbf{j}_{0} \cdot \mathbf{e}+\int_{\Omega_{c}} \mathbf{j}_{0} \cdot \partial_{t} \mathbf{a} \\
& =\int_{\Omega_{c}} \mathbf{j}_{0} \cdot \mathbf{e}+\int_{\Omega} \mathbf{t}_{0} \cdot \partial_{t} \mathbf{b} \\
& =R I+\partial_{t} \int_{\Omega} \mathbf{t}_{0} \cdot \mathbf{b}
\end{aligned}
$$

where $\mathbf{j}_{0}=$ curl $_{\mathbf{t}_{0}}$ (see the aforementioned works for details). However, by doing so, one forgets that the starting Equation (5) holds under the hypothesis that fields do not vary with time. In spite of the fact that the derivation of (6)-(8) is somehow simplistic, all these equations are found to be correct.

A completely different approach is to postulate that the current flows out of special thin "generator regions" $\Omega_{e m f, i}$, where a source of electromotive force exists [17,24-26]. Inside the generator regions, the electric field is conservative and hence (2) is well posed, hence the voltage between the electrodes of a generator writes:

$$
U=\int_{\gamma_{i}} \mathbf{e}_{\mathbf{i}} \cdot \mathbf{d} \mathbf{l}
$$

where $\gamma_{i}$ is a path that joins the electrodes and $\mathbf{e}_{\mathbf{i}}$ is the electric field inside the generator. In practice, the integral in (9) is never computed explicitly. Generators are removed from the computational domain so that their surfaces become new boundaries of the domain, over which appropriate boundary conditions must be imposed. Depending on the formulation, the voltage can be imposed either strongly (a formulation) or weakly (h formulation)—see details in [24,26].

This approach removes the practical difficulty of numerically defining voltages and has a correct power balance (all the electrical power which exits from generators enters in the domain), but it is not completely satisfying from the point of view of physics significance: voltage ought to be defined independently on how it is applied-that is, basing it exclusively on the electric field e inside the computational domain.

Finally, one observes that numerical modelling of the coupling of electrical circuits with computational domains is addressed in specific ways for each formulation. This gives rise to a large number of different formulas which express the link between global (currents and voltages) and local quantities (fields and potentials). This is a source of complication, including from the pedagogical point of view, because many different approaches ought to be introduced to students.

The purpose of this work is twofold: First, to clarify the notion of "voltage" in the case of time varying fields, and to expressing it by exclusively using the electric field inside the domain (without resorting to any potential), as it should be! Second, to devise a general method to write coupling equations between external electrical circuits and 2D/3D computational domains.

The article is organised as follows: an operator $\mathcal{U}_{i j}[\mathbf{e}]$ which expresses the voltage between two ports $i, j$ for a given electric field $\mathbf{e}$ will be defined. It will be demonstrated that, by using this definition, the power balance is intrinsically respected: this gives a precise, physical significance to the notion of "voltage", even in the case of time-varying fields (Section 2). As a side product, a similar operator $\mathcal{I}_{n}[\mathbf{h}]$ for the current is obtained. It will be observed that the classical definition of current (1) can be rewritten as a particular case of usage of the new operator (Section 3). Then, the existing scientific literature is analysed critically and the most used Finite Element formulations for coupling external circuits and computational domains are reviewed in Section 4. It will be demonstrated that all of them can be retrieved by using the newly introduced operators $\mathcal{U}_{i j}[\mathbf{e}]$ and $\mathcal{I}_{n}[\mathbf{h}]$. 
The obtained results are discussed in Section 5. Finally, the conclusions can be found in Section 6.

\section{Definition of Voltage}

In [27], Hiptmair and Sterz pointed out the difficulties to rigorously define what voltage is and suggested it could be defined through the notion of electric power. Based on this idea, it will be shown that it is possible to give a precise meaning to the notion of "voltage" in the case of time varying fields and for an arbitrary number of ports. The proposed definition of voltage respects the power balance and it is coherent with all previous works. Moreover, the proposed definition of voltage relies exclusively on the electric field; therefore, it is independent of any formulation or numerical method. To go further, the following theorem has to be demonstrated:

Theorem $\mathbf{1}$ (fake power). Let $\mathbf{e}_{1}$ and $\mathbf{h}_{2}$ be an electric and a magnetic field, defined in a domain $\Omega_{c}$ and $\Omega$, where $\Omega_{c} \subset \Omega$ is the conductive part of $\Omega$. Let $\mathbf{b}_{1}$ and $\mathbf{j}_{2}$ the magnetic flux density and the current density associated respectively with $\mathbf{e}_{1}$ and $\mathbf{h}_{2}$, and assume that displacement currents are neglected:

$$
\begin{aligned}
\operatorname{curl} \mathbf{e}_{1} & =-\partial_{t} \mathbf{b}_{1} \\
\operatorname{curl} \mathbf{h}_{2} & =\mathbf{j}_{2}
\end{aligned}
$$

Assume that the boundary $\partial \Omega=\Gamma_{h} \cup \Gamma_{e}$ can be parted in two surfaces $\Gamma_{e}$ and $\Gamma_{h}$ where the following boundary conditions hold:

$$
\begin{array}{lll}
\text { on } \Gamma_{e}: & \mathbf{n} \times \mathbf{e}_{1}=0 & \left(\Rightarrow \mathbf{n} \cdot \partial_{t} \mathbf{b}_{1}=0\right) \\
\text { on } \Gamma_{h}: & \mathbf{n} \times \mathbf{h}_{2}=0 & \left(\Rightarrow \mathbf{n} \cdot \mathbf{j}_{2}=0\right)
\end{array}
$$

and that there exists a couple of potentials $\left(\mathbf{a}_{1}, v_{1}\right)$ such that:

$$
\begin{gathered}
\mathbf{e}_{1}=-\partial_{t} \mathbf{a}_{1}-\operatorname{grad} v_{1} \\
\mathbf{n} \times\left.\partial_{t} \mathbf{a}_{1}\right|_{\Gamma_{e}}=0
\end{gathered}
$$

Under this hypothesis, one has:

1. The scalar potential $v_{1}$ is equipotential on each of the $N$ connected components $\Gamma_{e, n}$ of $\Gamma_{e} \cap \partial \Omega_{c}$ :

$$
\left.v_{1}\right|_{\Gamma_{e, n}}=V_{1, n}
$$

Moreover, for a given electric field $\mathbf{e}_{1}$, the set of potentials $\left\{V_{1, n}\right\}$ is unique up to an arbitrary constant value.

2. The following equality holds:

$$
\begin{aligned}
\mathcal{P}\left[\mathbf{e}_{1}, \mathbf{h}_{2}\right] & =\int_{\Omega_{c}} \mathbf{j}_{2} \cdot \mathbf{e}_{1}+\int_{\Omega} \mathbf{h}_{2} \cdot \partial_{t} \mathbf{b}_{1}=\int_{\Omega_{c}} \operatorname{curl} \mathbf{h}_{2} \cdot \mathbf{e}_{1}-\int_{\Omega} \mathbf{h}_{2} \cdot \operatorname{curl} \mathbf{e}_{1} \\
& =-\int_{\Omega_{c}} \mathbf{j}_{2} \cdot \operatorname{grad} v_{1}
\end{aligned}
$$

3. Let $I_{2, n}$ be the total current which enters in $\Omega_{c}$ through $\Gamma_{e, n}$ :

$$
I_{2, n}=-\int_{\Gamma_{e, n}} \mathbf{n} \cdot \mathbf{j}_{2}
$$

where $\mathbf{n}$ is the unitary vector normal to $\Gamma_{e, n}$ oriented outwardly. Then, the following equality holds:

$$
\mathcal{P}\left[\mathbf{e}_{1}, \mathbf{h}_{2}\right]=\sum_{n} V_{1, n} I_{2, n}
$$


Before giving the demonstration, some remarks are mandatory:

- It has to be highlighted that the hypothesis of the theorem does not require that constitutive laws are enforced (they could eventually be, but this is not necessary):

$$
\mathbf{j}_{2} \neq \sigma \mathbf{e}_{1} \quad ; \quad \mathbf{b}_{1} \neq \mu \mathbf{h}_{2}
$$

In this case, the $\mathrm{E}$ side and the $\mathrm{H}$ side of Tonti's diagram are unlinked, thus $\mathbf{e}_{1}$ and $\mathbf{h}_{2}$ cannot be both the true electric field $\mathbf{e}$ and magnetic fields $\mathbf{h}$ :

$$
\begin{array}{cccccccc}
\text { E-side: } & v_{1} & \stackrel{\text { grad }}{\longrightarrow} & \mathbf{e}_{1}, \mathbf{a}_{1} & \stackrel{\text { curl }}{\longrightarrow} & \mathbf{b}_{1} & \stackrel{\text { div }}{\longrightarrow} & 0 \\
& & & \vdots & & \vdots & & \\
\text { H-side: } & 0 & \stackrel{\text { div }}{\longleftarrow} & \mathbf{j}_{2} & \stackrel{\text { curl }}{\longleftarrow} & \mathbf{h}_{2} & &
\end{array}
$$

Hence, the quantity $\mathcal{P}\left[\mathbf{e}_{1}, \mathbf{h}_{2}\right]$ defined by (17), which is homogeneous to a power, has no physical significance. For this reason, in the general case, $\mathcal{P}\left[\mathbf{e}_{1}, \mathbf{h}_{2}\right]$ will be hereafter called "fake" power. Conversely, when constitutive laws are enforced, then $\mathbf{e}_{1}=\mathbf{e}$ and $\mathbf{h}_{2}=\mathbf{h}$ are the true electric and magnetic fields, and $\mathcal{P}[\mathbf{e}, \mathbf{h}]$ is the true, physically significant, instantaneous power which is provided to the domain by the generators.

- The boundary $\Gamma_{e}$ writes:

$$
\Gamma_{e}=\underbrace{\left(\bigcup_{n} \Gamma_{e, n}\right)}_{\subseteq \partial \Omega_{c}} \cup \Gamma_{b}
$$

The surfaces $\Gamma_{e, n}$ are the ports which connect the computational domain with the external electric circuit. As such, they are also part of the boundary of $\partial \Omega_{c}$, where the scalar potential $v_{1}$ is defined hence. The surface $\Gamma_{b}$ is the remaining part of $\Gamma_{e}$, where the scalar potential are not defined (Figure 1). The boundary of $\Omega_{c}$ writes:

$$
\partial \Omega_{c}=\left(\bigcup_{n} \Gamma_{e, n}\right) \cup \Gamma_{j}
$$

$\Gamma_{j}$ is the interface between $\Omega_{c}$ and the remaining (insulator) part of $\Omega$, where $\mathbf{n} \cdot \mathbf{j}=0$.

Proof of Theorem 1. By writing grad $v_{1}=-\mathbf{e}_{1}-\mathbf{a}_{1}$ and by using (15) on each port $\Gamma_{e, n}$ one has:

$$
\mathbf{n} \times\left.\operatorname{grad} v_{1}\right|_{\Gamma_{e, n}}=-\mathbf{n} \times\left.\partial_{t} \mathbf{a}_{1}\right|_{\Gamma_{e, n}}-\mathbf{n} \times\left.\mathbf{e}_{1}\right|_{\Gamma_{e, n}}=0
$$

Hence, $v_{1}$ is constant on each port $\Gamma_{e, n}$. By using (14), the first integral of (17) writes:

$$
\int_{\Omega_{c}} \mathbf{j}_{2} \cdot \mathbf{e}_{1}=-\int_{\Omega_{c}} \mathbf{j}_{2} \cdot \partial_{t} \mathbf{a}_{1}-\int_{\Omega_{c}} \mathbf{j}_{2} \cdot \operatorname{grad} v_{1}
$$

By integrating by parts, the second integral of (17) writes:

$$
\begin{aligned}
\int_{\Omega} \mathbf{h}_{2} \cdot \partial_{t} \mathbf{b}_{1} & =\int_{\Omega} \mathbf{h}_{2} \cdot \partial_{t} \operatorname{curl} \mathbf{a}_{1} \\
& =\int_{\Omega} \mathbf{j}_{2} \cdot \partial_{t} \mathbf{a}_{1}+\int_{\partial \Omega} \mathbf{h}_{2} \cdot \mathbf{n} \times \partial_{t} \mathbf{a}_{1} \\
& =\int_{\Omega} \mathbf{j}_{2} \cdot \partial_{t} \mathbf{a}_{1}
\end{aligned}
$$

where the boundary integral vanishes due to the boundary conditions (12) and (13). By using together (26) and (27), one obtains (18). Finally, by using integration by parts, (18) writes:

$$
-\int_{\Omega_{c}} \mathbf{j}_{2} \cdot \operatorname{grad} v_{1}=\int_{\Omega_{c}} v_{1} \operatorname{div} \mathbf{j}_{2}-\int_{\partial \Omega_{c}} v_{1} \mathbf{n} \cdot \mathbf{j}_{2}
$$




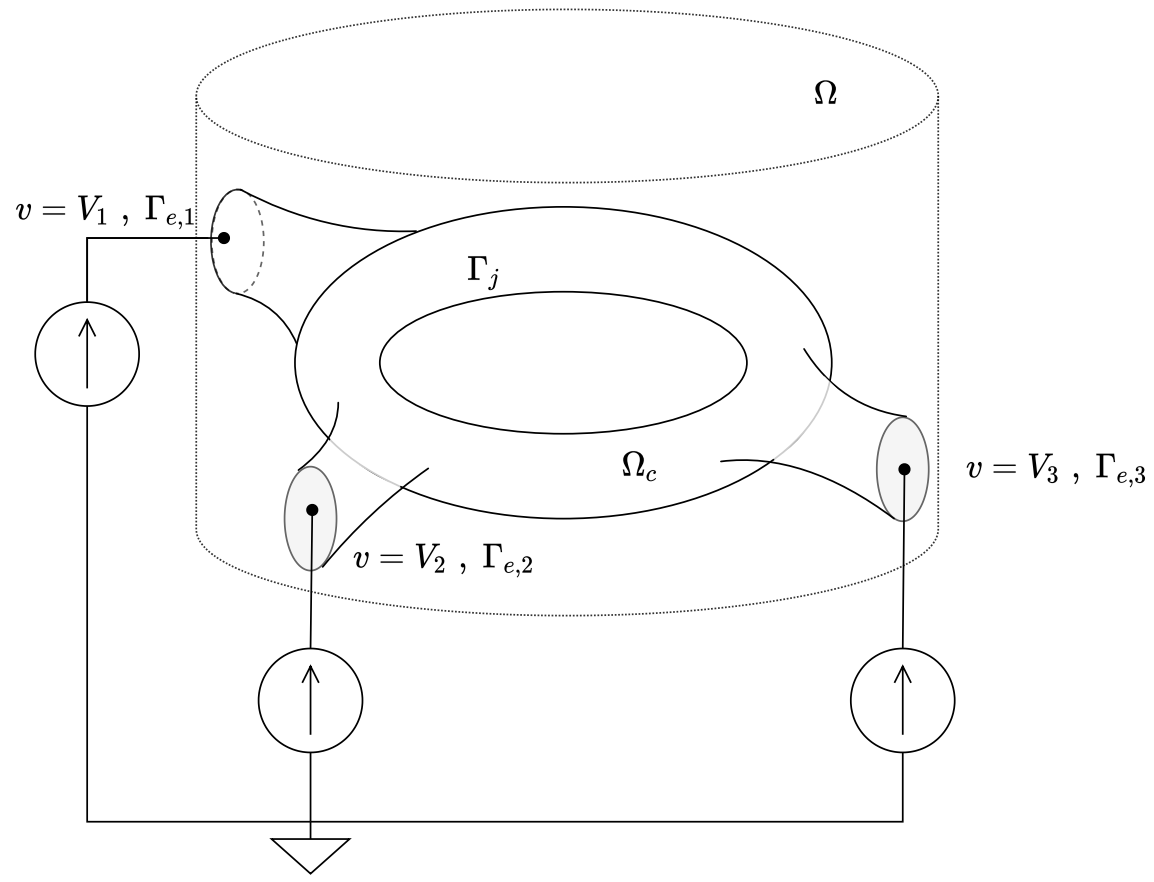

Figure 1. Computational domain $\Omega$ composed of a conductive part $\Omega_{c}$. The generators feed the conductive part through the three ports $\Gamma_{e, 1 \ldots 3}$. The boundary of $\Omega_{c}$ is composed of the ports $\Gamma_{e, 1 \ldots 3}$ and of $\Gamma_{j}$.

The first integral on the right-hand side vanishes because $\operatorname{div} \mathbf{j}_{2}=\operatorname{div} \operatorname{curl} \mathbf{h}_{2}=0$. The boundary of $\Omega_{\mathcal{C}}$ can be parted as:

$$
\partial \Omega_{c}=\left(\bigcup_{n} \Gamma_{e, n}\right) \cup \Gamma_{j}
$$

where $\Gamma_{j}$ is the interface between the conductive and insulator part of the domain $\Omega_{i}=$ $\Omega \backslash \Omega_{c}$. The current cannot flow in or out from $\Gamma_{j}$, that is:

$$
\left.\mathbf{n} \cdot \mathbf{j}_{2}\right|_{\Gamma_{j}}=0
$$

hence the boundary integral in (28) writes:

$$
\mathcal{P}\left[\mathbf{e}_{1}, \mathbf{h}_{2}\right]=\sum_{n}-\int_{\Gamma_{e, n}} v_{1} \mathbf{n} \cdot \mathbf{j}_{2}=\sum_{n} V_{n}\left(-\int_{\Gamma_{e, n}} \mathbf{n} \cdot \mathbf{j}_{2}\right)=\sum_{n} V_{n} I_{n}
$$

Finally, the uniqueness of the set $\left\{V_{1, n}\right\}$ can be demonstrated by observing that these values depend uniquely on the electric field $\mathbf{e}_{1}$. Hence, a set of $N-1$ independent equations can be obtained from (31) by selecting an arbitrary port $\Gamma_{e, r e f}$ (namely $\Gamma_{e, r e f}=\Gamma_{e, N}$ ) and by considering $N-1$ magnetic fields $\mathbf{h}_{2, n}$ such that $I_{2, \text { ref }}=-I_{2, n}=1 \mathrm{~A}$.

$$
\begin{cases}V_{1,1}-V_{1, \text { ref }} & =\mathcal{P}\left[\mathbf{e}_{1}, \mathbf{h}_{2,1}\right] \\ V_{1,2}-V_{1, \text { ref }} & =\mathcal{P}\left[\mathbf{e}_{1}, \mathbf{h}_{2,2}\right] \\ \vdots & \\ V_{1, N-1}-V_{1, \text { ref }} & =\mathcal{P}\left[\mathbf{e}_{1}, \mathbf{h}_{2, N-1}\right]\end{cases}
$$


In order to define the voltage $U_{i j}$ between two ports $\Gamma_{e, i}$ and $\Gamma_{e, j}$, the set $\mathcal{H}_{i j}$ of "test" magnetic field is defined:

$$
\mathcal{H}_{i j}=\left\{\mathbf{h}^{\prime} \in \mathbf{H}(\operatorname{curl}, \Omega): \mathbf{n} \times \mathbf{h}^{\prime}=0 \text { on } \Gamma_{h}, I_{i}^{\prime}=-I_{j}^{\prime}=1 \mathrm{~A}, I_{n \neq i, j}^{\prime}=0\right\}
$$

where $I_{n}^{\prime}$ is the net current due to $\mathbf{j}^{\prime}=\operatorname{curl} \mathbf{h}^{\prime}$ which enters in $\Omega$ through the $n^{\text {th }}$ port $\Gamma_{e, n}$. The following theorem provides a well-posed definition of the voltage $U_{i j}$ :

Theorem 2 (definition of voltage). Let $\mathbf{e}$ be the electric field in $\Omega_{c}$, and $\Gamma_{e, i}$ and $\Gamma_{e, j}$ two ports. There is one and only one value $U_{i j}[\mathbf{e}]$ such that:

$$
U_{i j}[\mathbf{e}]=\frac{\mathcal{P}\left[\mathbf{e}, \mathbf{h}^{\prime}\right]}{1 A}
$$

for any $\mathbf{h}^{\prime} \in \mathcal{H}_{i j}$.

Proof of Theorem 2. Theorem 1 holds with $\mathbf{e}_{1}=\mathbf{e}$ and $\mathbf{h}_{2}=\mathbf{h}^{\prime}, \forall \mathbf{h}^{\prime} \in \mathcal{H}_{i j}$, and thus the fake power writes:

$$
\mathcal{P}\left[\mathbf{e}, \mathbf{h}^{\prime}\right]=V_{i} I_{i}^{\prime}+V_{j} I_{j}^{\prime}=\left(V_{i}-V_{j}\right) \times 1 \mathrm{~A}
$$

In order to demonstrate that this value is unique, it must be shown that it does not depend on $\mathbf{h}^{\prime}$ nor on the gauge of $(\mathbf{a}, v)$. The left-hand side term of (35) does not depend on the gauge because of (17), and the right-hand side of (35) does not depend on $\mathbf{h}^{\prime}$. Hence, $\mathcal{P}\left[\mathbf{e}, \mathbf{h}^{\prime}\right]$ is independent of both $\mathbf{h}^{\prime}$ and the gauge of $(\mathbf{a}, v)$. Therefore, $\mathcal{P}\left[\mathbf{e}, \mathbf{h}^{\prime}\right]$ depends exclusively on the electric field $\mathbf{e}$, which proves its uniqueness. Hence, $U_{i j}[\mathbf{e}]=V_{i}-V_{j}$ is the only value which satisfies (34) whatever $\mathbf{h}^{\prime} \in \mathcal{H}_{i j}$.

This definition of voltage is well posed because $U_{i j}$ depends exclusively on the electric field $\mathbf{e}$, and it holds as well for static and time varying fields. One observes that when $V_{n}=\left.v\right|_{\Gamma_{e, n}}$ are the voltages of generators that feed the device (Figure 1), and $\mathbf{e}_{1}=\mathbf{e}$ and $\mathbf{h}_{2}=\mathbf{h}$ are the true electric and magnetic fields, the power balance is verified in that (20) writes:

$$
\mathcal{P}[\mathbf{e}, \mathbf{h}]=\int_{\Omega_{c}} \mathbf{j} \cdot \mathbf{e}+\int_{\Omega} \mathbf{h} \cdot \partial_{t} \mathbf{b}=\sum_{n} V_{n} I_{n}
$$

This result is important because it provides a precise physical significance to "voltages", that is the unique set of values $U_{i j}=V_{i}-V_{j}$ such that the power balance (36) is respected. In particular, in the case of a simple two-ports domain, the voltage can be defined like the ratio between the instantaneous power injected into the domain and the instantaneous current. One observes that the electric vector potential $\mathbf{t}_{0}$ defined beforehand belongs to $\mathcal{H}_{i j}$ : this shows that indeed (5) and (7) are particular cases of (34).

\section{Expression of the Current}

Now that a precise definition has been given to the term "voltage", one observes that the roles of $\mathbf{e}_{1}$ and $\mathbf{h}_{2}$ can be exchanged, so as to provide a useful expression of the net current which enters in the domain through electrodes. To this aim, let us define the set $\mathcal{E}_{n}$ of "test" electric fields:

$$
\begin{array}{r}
\mathcal{E}_{n}=\left\{\mathbf{e}^{\prime} \in \mathbf{H}\left(\operatorname{curl}, \Omega_{c}\right): \mathbf{n} \times \mathbf{e}^{\prime}=0 \text { on } \Gamma_{e}, \mathbf{e}^{\prime}=-\partial_{t} \mathbf{a}^{\prime}-\operatorname{grad} v^{\prime}\right. \\
\text { with } \left.v^{\prime} \equiv 1 \mathrm{~V} \text { on } \Gamma_{e, n}, v^{\prime} \equiv 0 \text { on } \Gamma_{e, \neq n}\right\}
\end{array}
$$

The following theorem provides a useful formula to compute the electric current which enters in the domain through a port: 
Theorem 3 (computation of current). Let $\mathbf{h}$ be the magnetic field in $\Omega$, and $\Gamma_{e, n}$ a port. The electric current $I_{n}$, which enters in the domain through $\Gamma_{e, n}$, is equal to:

$$
I_{n}=\frac{\mathcal{P}\left[\mathbf{e}^{\prime}, \mathbf{h}\right]}{1 \mathrm{~V}}
$$

for any $\mathbf{e}^{\prime} \in \mathcal{E}_{n}$.

Proof of Theorem 3. Theorem 1 holds with $\mathbf{e}_{1}=\mathbf{e}^{\prime}$ and $\mathbf{h}_{2}=\mathbf{h}, \forall \mathbf{e}^{\prime} \in \mathcal{E}_{n}$, thus the fake power writes:

$$
\mathcal{P}\left[\mathbf{e}^{\prime}, \mathbf{h}\right]=\left.v^{\prime}\right|_{\Gamma_{e, n}} \times I_{n}=1 \mathrm{~V} \times I_{n}
$$

In this case, there is no need to prove the uniqueness of $I_{n}$ because currents are correctly defined. Nevertheless, an argument similar to the one which has been used to prove Theorem 2 applies as well (that is, $\mathcal{P}\left[\mathbf{e}^{\prime}, \mathbf{h}\right]$ does not depend on the gauge, $I_{n}$ does not depend on $\mathbf{e}^{\prime}$ ).

One observes that even if (38) expresses the current based on $\mathbf{h}$, only its curl matters:

$$
I_{n}=-\int_{\Gamma_{e, n}} \mathbf{n} \cdot \mathbf{j}=-\int_{\Gamma_{e, n}} \mathbf{n} \cdot \operatorname{curl} \mathbf{h}=\frac{\mathcal{P}\left[\mathbf{e}^{\prime}, \mathbf{h}\right]}{1 \mathrm{~V}}
$$

Finally, one observes that even the classical definition of current (1) can be rewritten as a particular case of (38). In fact, consider as test electric field the gradient of a scalar potential $\mathbf{e}^{\prime}=-\operatorname{grad} v^{\prime}$, the support of which is a thin layer of thickness $w$ which lays on the surface $\Sigma$. Assume that $v^{\prime} \equiv 1 \mathrm{~V}$ on $\Sigma$. In the limit of $w \rightarrow 0$, the electric field writes $\mathbf{e}^{\prime}=(-\mathbf{n}) \delta_{\Sigma}$, where $\delta_{\Sigma}$ is the Dirac distribution associated with the surface $\Sigma$. Hence, by using this particular test electric field with (38), the current writes:

$$
I=\frac{\mathcal{P}\left[\mathbf{e}^{\prime}, \mathbf{h}\right]}{1 \mathrm{~V}}=\int_{\Omega_{c}} \mathbf{j} \cdot(-\mathbf{n}) \delta_{\Sigma}=-\int_{\Sigma} \mathbf{n} \cdot \mathbf{j}
$$

\section{Formulations}

Hereafter, several Finite Element formulations are reviewed with the purpose of showing that, for all of them, the coupling equations with electrical circuits can be easily derived by using (34) and (38). In order to somehow simplify the notation hereafter, the divisions by $1 \mathrm{~A}$ or $1 \mathrm{~V}$ will be dropped and the expressions of the voltage and current will write simply:

$$
\begin{array}{ll}
\mathcal{U}_{i j}[\mathbf{e}]: \mathbf{e} \mapsto \mathcal{P}\left[\mathbf{e}, \mathbf{h}^{\prime}\right] \quad \forall \mathbf{h}^{\prime} \in \mathcal{H}_{i j} \\
\mathcal{I}_{n}[\mathbf{h}]: \mathbf{h} \mapsto \mathcal{P}\left[\mathbf{e}^{\prime}, \mathbf{h}\right] \quad \forall \mathbf{e}^{\prime} \in \mathcal{E}_{n}
\end{array}
$$

For each formulation, particular test fields $\mathbf{h}^{\prime} \in \mathcal{H}_{i j}$ or $\mathbf{e}^{\prime} \in \mathcal{E}_{n}$ will be chosen in order to simplify the writing of formulations at the discrete level. The purpose of this section is to show that known expressions to compute voltages and currents can be viewed as particular cases of (34) and (38), respectively.

\subsection{Electrokinetics}

The scalar potential electrokinetic formulation writes:

$$
\operatorname{div}(\sigma \operatorname{grad} v)=0
$$

that is $\operatorname{div} \mathbf{j}=0$, with $\mathbf{e}=-\operatorname{grad} v$, with $\sigma=$ electrical conductivity. The weak form writes:

$$
\int_{\Omega_{c}} \sigma \operatorname{grad} v \cdot \operatorname{grad} v^{\prime}+\int_{\partial \Omega_{c}}(\mathbf{n} \cdot \mathbf{j}) \cdot v^{\prime}=0
$$


where $v$ and $v^{\prime}$ belong to appropriate function spaces.

At the discrete level, the domain $\Omega=\Omega_{c}$ is meshed and the set of nodes $\mathbb{N}$ is parted as:

$$
\mathbb{N}=\left[\bigcup_{n} \mathbb{N}_{i, n}\right] \cup \mathbb{N}_{v}
$$

where $\mathbb{N}_{i, n}$ is the set of nodes on the $n^{\text {th }}$ port $\Gamma_{e, n}$, and $\mathbb{N}_{v}$ is the set of the remaining nodes. Classical nodal shape functions $s_{n}(\mathbf{x})$ are associated with each node of $\mathbb{N}_{v}$, whereas special isopotential shape functions $f_{n}(\mathbf{x})$ are associated with each port $\Gamma_{e, n}$ [24-26] (see Figure 4 of [25]):

$$
f_{n}(\mathbf{x})=\sum_{k \in \mathbb{N}_{i, n}} v_{k}(\mathbf{x})
$$

The support of $f_{n}$ is bound to the layer of elements which touch $\Gamma_{e, n}$. One observes that $f_{n} \equiv 1$ on $\Gamma_{e, n}$ and $f_{n} \equiv 0$ on any other port, thus $\mathbf{e}_{n}^{\prime}=-\operatorname{grad} f_{n} \in \mathcal{E}_{n}$. By using (38) the net current $I_{n}$ which enters in $\Omega_{c}$ through the $n^{\text {th }}$ writes:

$$
I_{n}=\int_{\Omega_{c}} \mathbf{j} \cdot\left(-\operatorname{grad} f_{n}\right)=\int_{\Omega_{c}} \sigma \operatorname{grad} v \cdot \operatorname{grad} f_{n}=-\int_{\partial \Omega_{c}}(\mathbf{n} \cdot \mathbf{j}) \cdot f_{n}
$$

where the last equality comes from (45). One observes that the latter equation writes

$$
I_{n}=\mathcal{I}_{n}[\mathbf{h}]=\mathcal{P}\left[-\operatorname{grad} f_{n}, \mathbf{h}\right]
$$

where $\mathbf{h}$ is the magnetic field (which is not computed by using this formulation) and $\mathbf{j}=$ curl h.

The discrete approximation of $v$ writes:

$$
v(\mathbf{x})=\sum_{n \in \mathbb{N}_{v}} v_{n} s_{n}(\mathbf{x})+\sum_{k} V_{k} f_{k}(\mathbf{x})
$$

With this formulation, the potential $V_{n}$ on the port $\Gamma_{e, n}$ can be imposed strongly, as it belongs directly to the set of unknowns. When Galerkin's method is used, the same shape functions are used both to expand the unknowns and as test functions; hence, the coupling equation which allows to impose the current $I_{n}$ appears naturally for $v^{\prime}=f_{n}$ :

$$
\int_{\Omega_{c}} \sigma \operatorname{grad} v \cdot \operatorname{grad} f_{n}-I_{n}=0
$$

In order to illustrate this formulation with a practical example, consider a conductor $(\sigma=10 \mathrm{~S} / \mathrm{m})$ with a hole and a low conductive region $\left(\sigma=10^{-6} \mathrm{~S} / \mathrm{m}\right)$, where a continuous voltage of $5 \mathrm{~V}$ is imposed between the two electrodes on the left and on the right (Figure 2a). A test electric field $\mathbf{e}^{\prime}$ is computed by solving the electrokinetic formulation with a random conductivity (Figure $2 \mathrm{~b}$ ). A voltage of $1 \mathrm{~V}$ is imposed between the electrodes. A realisation of the test electric field $\mathbf{e}^{\prime}$ is depicted in Figure 2c, while the true current density $\mathbf{j}$ is depicted in Figure 2d. The total current is computed by using the classical expressions (1) and (49). It is observed that the results obtained by the two expressions converge to the same value as the mesh is refined (Table 1). The corresponding program is written in the FreeFem++ environment [28], and it is freely available at https://github.com/scorretti/examples_ circuit_coupling (accessed on 23 November 2021). 
Table 1. Current computed by using (1) and (49) for several refinements of the mesh.

\begin{tabular}{ccc}
\hline Number of Elements & $\boldsymbol{I}=-\int_{\boldsymbol{\Sigma}} \mathbf{n} \cdot \mathbf{j}$ & $\boldsymbol{I}=\int_{\boldsymbol{\Omega}_{c}} \mathbf{j} \cdot \mathbf{e}^{\prime}$ \\
\hline 1030 & 12.4184 & 11.7614 \\
9462 & 12.3455 & 12.2821 \\
25,852 & 12.3379 & 12.3383 \\
104,109 & 12.3337 & 12.3184 \\
233,938 & 12.3326 & 12.3268 \\
415,283 & 12.3321 & 12.3367 \\
\hline
\end{tabular}

a)

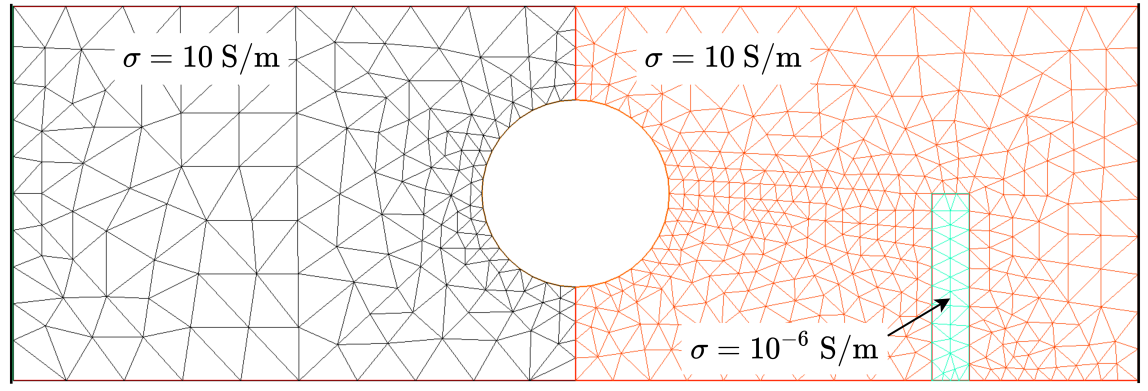

b)
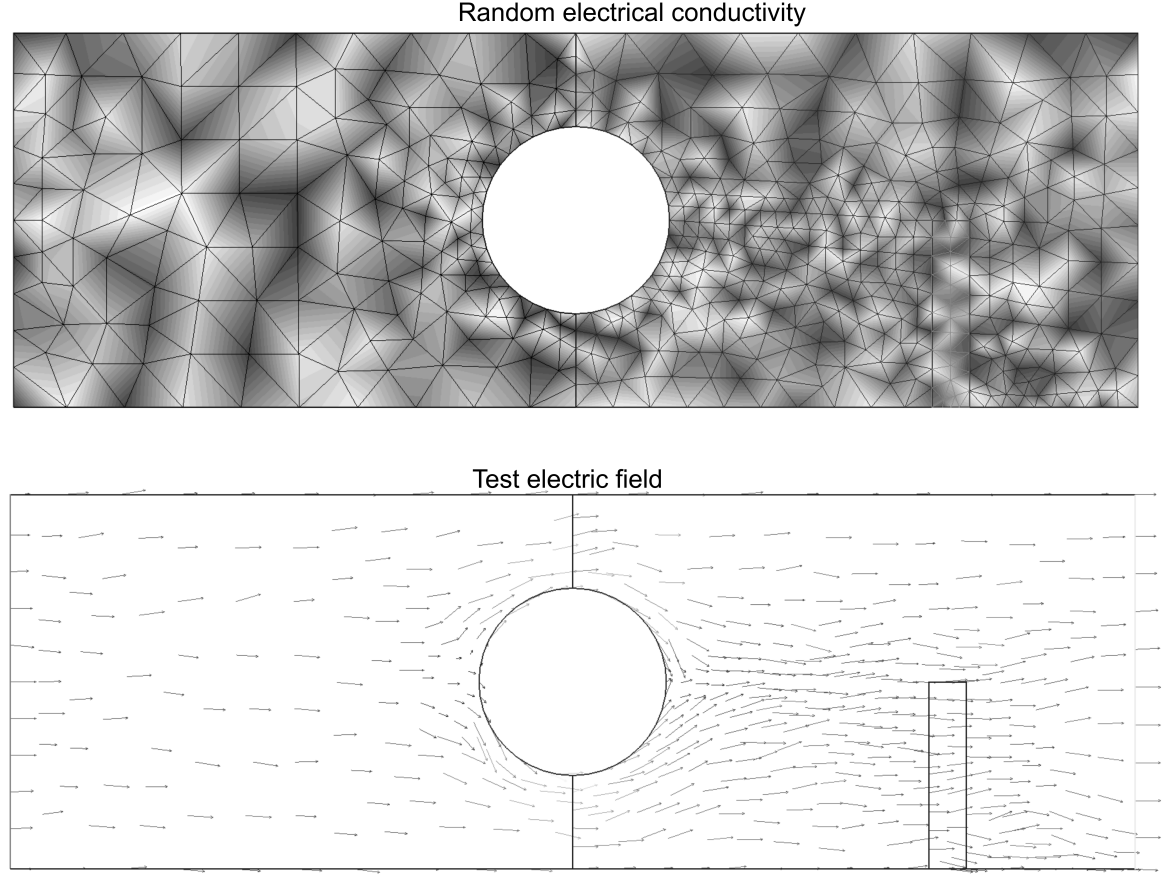

d)

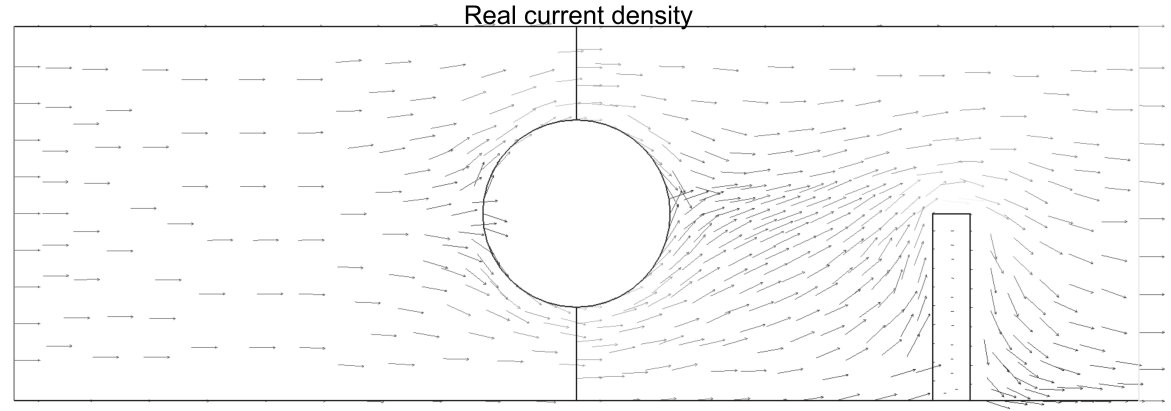

Figure 2. The domain composed of a conductor with a poorly conductive region (green) and a hole (a). A random conductivity (b) is used to compute the test electric field $\mathbf{e}^{\prime}$ (c) that, on its turn, is used to compute the total current $I$ corresponding to the real current density (d). 


\subsection{Eddy Current Formulations}

A very large number of formulations have been developed to model eddy currents. In general, either the current or the voltage can be imposed strongly, whereas the other quantity has to be imposed weakly. Hereafter, some of the most used formulations are reviewed, but similar considerations can be taken on for any other formulation.

\subsection{1. $\mathbf{t}-\mathbf{t}_{0}-\phi$ Formulation}

With the $\mathbf{t}-\mathbf{t}_{0}-\phi$ formulation, the equations on the $\mathrm{E}$ side of Tonti's diagram (curl $\mathbf{e}=-\partial_{t} \mathbf{b}, \operatorname{div} \mathbf{b}=0$ ) are imposed weakly:

$$
\operatorname{curl}(\rho \mathbf{j})=-\partial_{t}(\nu \mathbf{h})
$$

with $\rho=1 / \sigma=$ electrical resistivity and $v=1 / \mu=$ magnetic reluctivity. The magnetic field $\mathbf{h}$ and the current density $\mathbf{j}$ write, respectively $[13,23,29]$ :

$$
\begin{gathered}
\mathbf{h}= \begin{cases}\mathbf{t}+\sum_{n} I_{n} \mathbf{t}_{0, n}-\operatorname{grad} \phi & \text { in } \Omega_{c} \\
\sum_{n} I_{n} \mathbf{t}_{0, n}-\operatorname{grad} \phi & \text { in } \Omega \backslash \Omega_{c}\end{cases} \\
\mathbf{j}=\operatorname{curl} \mathbf{t}+\sum_{n} I_{n} \mathbf{t}_{0, n} \text { in } \Omega_{c}
\end{gathered}
$$

where $\phi$ is a magnetic scalar potential and $\mathbf{t}_{0, n}$ are precomputed electric vector potentials defined on $\Omega_{0} \supset \Omega_{c}$. For each two-ports solid conductor, a potential $\mathbf{t}_{0, n}$ is precomputed in such a way that the net current associated with $\mathbf{j}_{0, n}=$ curl $\mathbf{t}_{0, n}=1$ A (see Figure 5.29, p. 171 of [29]), thus $\mathbf{t}_{0, n} \in \mathcal{H}_{n}$ (in order to simplify the notation, where two-ports cases are concerned, one writes $n$ instead of $i, j$ ).

Currents in coils $I_{n}$ belong to the set of unknowns and are, therefore, imposed strongly. By taking $\mathbf{h}^{\prime}=\mathbf{t}_{0, n}$, one finds with (34) the expression of the voltage $U_{n}$ associated with each coil:

$$
U_{n}=\mathcal{P}\left[\mathbf{e}, \mathbf{t}_{0, n}\right]=\int_{\Omega_{c}} \mathbf{j}_{0, n} \cdot \mathbf{e}+\int_{\Omega_{0}} \mathbf{t}_{0, n} \cdot \partial_{t} \mathbf{b}
$$

In the case of nonsimply connected conductors, additional potentials $\mathbf{t}_{0, k}$ and associated unknowns $I_{k}$ must be added to cope with the so-called connexity problem (that is, to be able to impose Ampère's theorem). The corresponding voltages $U_{k}$ are set to 0 by using (55):

$$
U_{k}=\int_{\Omega_{c}} \mathbf{j}_{0, k} \cdot \mathbf{e}+\int_{\Omega_{0}} \mathbf{t}_{0, k} \cdot \partial_{t} \mathbf{b}=0
$$

By the way, one observes that in the case of static fields $\left(\partial_{t} \equiv 0\right)$ one seamlessly obtains the dual electrokinetic formulation [30], with respect of the aforementioned $v$ formulation, by imposing curl $\mathbf{e}=0$ :

$$
\operatorname{curl}\left[\rho \operatorname{curl}\left(\mathbf{t}+\sum_{n} \mathbf{t}_{0, n} I_{n}\right)\right]=0
$$

Voltages can be expressed by (34) as:

$$
U_{n}=\mathcal{P}\left[\mathbf{e}, \mathbf{t}_{0, n}\right]=\int_{\Omega_{c}} \mathbf{j}_{0, n} \cdot \mathbf{e}
$$

\subsection{2. $\mathbf{a}-v$ Formulation}

With the $\mathbf{a}-v$ formulation, the equations on the $\mathrm{H}$ side of Tonti's diagram (curl $\mathbf{h}=\mathbf{j}$, $\operatorname{div} \mathbf{j}=0$ ) are imposed weakly:

$$
\operatorname{curl}(\nu \mathbf{b})=\sigma \mathbf{e}
$$

The electric field $\mathbf{e}$ and the flux density $\mathbf{b}$ write, respectively

$$
\mathbf{e}=-\partial_{t} \mathbf{a}-\operatorname{grad} v \quad \text { in } \Omega_{c}
$$




$$
\mathbf{b}=\operatorname{curl} \mathbf{a}
$$

where $\mathbf{a}$ and $v$ are, respectively, a magnetic vector potential and an electric scalar potential. By using (34) together with (20), one observes that voltages is determined by imposing the scalar potential $v$ only. In fact, for any $\mathbf{h}^{\prime} \in \mathcal{H}_{i j}$ one has:

$$
U_{i j}=\mathcal{P}\left[\mathbf{e}, \mathbf{h}^{\prime}\right]=V_{i}-V_{j}
$$

For instance, in [25], this is accomplished by discretising the scalar potential $v$ as:

$$
v=\sum_{n} V_{i} v_{0, n}(\mathbf{x})
$$

where the function $v_{0, n}$ is any potential which corresponds to a voltage of $1 \mathrm{~V}$ for the $n^{\text {th }}$ solid conductor, and hence - grad $v_{0, n} \in \mathcal{E}_{n}$ (in [25], only two-ports conductors are considered). It is found that the current $I_{n}$ which flows through the $\mathrm{n}^{\text {th }}$ conductor writes:

$$
I_{n}=\int_{\Omega_{c}}-\sigma\left(\partial_{t} \mathbf{a}+\operatorname{grad} v\right) \cdot\left(-\operatorname{grad} v_{0, n}\right)=\int_{\Omega_{c}} \mathbf{j} \cdot \mathbf{e}^{\prime}
$$

where $\mathbf{e}^{\prime}=-\operatorname{grad} v_{0, n}$. It seems that, in order to retrieve (38), the term $\int_{\Omega} \mathbf{h} \cdot \partial_{t} \mathbf{b}$ is missing. However, in fact, notice that $-\operatorname{grad} v_{0, n}$ is defined only on $\Omega_{c}$ and it is curl free. It is possible to find a curl-free extension of $-\operatorname{grad} v_{0, n}$ over all the domain:

$$
\mathbf{e}^{\prime}= \begin{cases}-\operatorname{grad} v_{o, n} & \text { in } \Omega_{c} \\ -\operatorname{grad} \tilde{v} & \text { in } \Omega \backslash \Omega_{c}\end{cases}
$$

where $\tilde{v}$ is any scalar potential which enforce the continuity with $v$ on $\partial \Omega_{c}$. The thereby defined test electric field $\mathbf{e}^{\prime}$ is curl free and it is defined over all the domain; thus:

$$
\partial_{t} \mathbf{b}^{\prime}=-\operatorname{curl} \mathbf{e}^{\prime} \equiv 0
$$

Hence, the integral $\int_{\Omega} \mathbf{h} \cdot \partial_{t} \mathbf{b}^{\prime}$ vanishes and the expression (38) is found again. Notice that the real electric field $\mathbf{e}$ is not curl free, but test electric fields $\mathbf{e}^{\prime}$ are allowed to.

\subsection{3. $\mathbf{h}-\phi$ Formulation}

With the $\mathbf{h}-\phi$ formulation, the equations on the E side of Tonti's diagram (curl $\mathbf{e}=-\partial_{t} \mathbf{b}$, div $\mathbf{b}=0$ ) are imposed weakly. The computational domain is parted as $\Omega=\Omega_{c} \cup \Omega_{c}^{C}$ and a number of "cuts" have to be conducted to make $\Omega_{c}^{C}$ (insulators) simply connected. Assume that only two-ports solid conductors are present, some of which are coils. The field is impressed by generators through thin sections in the coils. The weak form of the formulation writes [17]:

$$
\int_{\Omega} \partial_{t}(\mu \mathbf{h}) \cdot \mathbf{h}^{\prime}+\int_{\Omega_{c}} \rho \operatorname{curl} \mathbf{h} \cdot \operatorname{curl} \mathbf{h}^{\prime}+\int_{\Gamma_{e}} \mathbf{n} \times \mathbf{e}_{s} \cdot \mathbf{h}^{\prime}=0
$$

The unknown of this formulation is, directly, the magnetic field $\mathbf{h}$, which is discretised as:

$$
\mathbf{h}=\sum_{k \in \mathbb{E}_{c}} h_{k} \mathbf{w}_{k}(\mathbf{x})+\sum_{n \in \mathbb{N}_{C}^{C}} \phi_{n} \mathbf{v}_{n}(\mathbf{x})+\sum_{n \in \mathbb{C}} I_{n} \mathbf{c}_{n}(\mathbf{x})
$$

where $\mathbb{E}_{c}$ is the set of edges internal to $\Omega_{c}, \mathbb{N}_{c}^{C}$ is the set of nodes on $\Omega_{c}^{C}$ and on its boundary, and $\mathbb{C}$ is the set of cuts required to make $\Omega_{c}^{C}$ simply connected (see $[17,24,31]$ for details). The key point is that $\mathbf{j}_{n}=\operatorname{curl} \mathbf{c}_{n}$ is a current density which corresponds to a unit net current in the ${ }^{\text {th }}$ coil, and hence $\boldsymbol{c}_{n} \in \mathcal{H}_{n}$. The coefficients $I_{n}$ represent the currents which 
flow through the $n^{\text {th }}$ coil, which can therefore be imposed strongly. It is found [17] that the corresponding voltage $U_{n}$ writes:

$$
U_{n}=\int_{\Omega} \partial_{t}(\mu \mathbf{h}) \cdot \mathbf{c}_{n}+\int_{\Omega_{c}} \rho \operatorname{curl} \mathbf{h} \cdot \operatorname{curl} \mathbf{c}_{n}=\mathcal{P}\left[\mathbf{e}, \mathbf{h}^{\prime}\right]
$$

where the test magnetic field is $\mathbf{h}^{\prime}=\mathbf{c}_{n}$ (see Figure 2 of [26]).

\subsection{Stranded Coils}

The case of stranded coils, composed of a multitude of thin insulated wires where eddy currents in individual wires can be neglected, is considerably simpler because the direction of $\mathbf{j}$ is known in advance. These kinds of coils are handled through a wire density vector $\mathbf{j}_{0}$ which corresponds to a unit current. The homogenised current density in stranded coils writes:

$$
\mathbf{j}=\mathbf{j}_{0} I
$$

where $\left\|\mathbf{j}_{0}\right\|=n_{c} / S_{c}$, with $n_{c}=$ number of turns, and $S_{c}=$ section of the coil [7]. Regardless of the formulation used, it is natural to impose currents which flow in stranded coils because the variable $I$ appears explicitly in (70). Voltage in stranded coils writes:

$$
U=R I+\partial_{t} \Psi
$$

where the resistance $R$ is known. Depending on the used formulation, the linkage flux $\Psi$ may be written in different ways $[7,12,26]$ :

$$
\Psi=\int_{\Omega_{c}} \mathbf{j}_{0} \cdot \mathbf{a}=\int_{\Omega} \mathbf{t}_{0} \cdot \mathbf{b}=\int_{\Omega} \mu \mathbf{h} \cdot \mathbf{h}_{s}
$$

where curl $\mathbf{t}_{0}=\operatorname{curl} \mathbf{h}_{s}=\mathbf{j}_{0}$ (see the aforementioned works for details). By observing that $R I=\int_{\Omega_{c}} \mathbf{j}_{0} \cdot \mathbf{e}$ and by using the latter equation, (71) boils up to one of (6) and (7), which are particular cases of (34) with $\mathbf{h}^{\prime}=\mathbf{t}_{0}$ or $\mathbf{h}^{\prime}=\mathbf{h}_{s}$ (cf. end of Section 2).

\section{Discussion}

In this work, an original couple of operators $\mathcal{U}_{i j}[\mathbf{e}]$ and $\mathcal{I}_{n}[\mathbf{h}]$ are introduced. These operators provide a rigorous definition of voltages and currents in terms of the electric and magnetic field only.

The notion of voltage is analysed and a general physical interpretation is given based on the electrical power balance of the computational domain. In the static case, the classical definition of voltage (2) is obtained as a particular case of (34), by taking as test magnetic field $\mathbf{h}_{\gamma}^{\prime}$, the Biôt-Savart field generated by a unit current which flows along the path $\gamma$ :

$$
U_{i j}=\int_{\Omega_{c}} \operatorname{curl} \mathbf{h}_{\gamma}^{\prime} \cdot \mathbf{e}+\int_{\Omega} \mathbf{h}_{\gamma}^{\prime} \cdot \partial_{t} \mathbf{b}=\int_{\Omega_{c}} \mathbf{j}_{\gamma}^{\prime} \cdot \mathbf{e}=\int_{\gamma} \mathbf{e} \cdot \mathbf{d} \mathbf{l}=V_{i}-V_{j}
$$

In the case of time varying fields, by taking the same test magnetic field $\mathbf{h}_{\gamma}^{\prime}$, the integral $\int_{\Omega} \mathbf{h}_{\gamma}^{\prime} \cdot \partial_{t} \mathbf{b}$ remains but the voltage can still be expressed as the difference of the scalar potential on the two (isopotential) ports, provided that the hypothesis of Theorem 1 is respected:

$$
U_{i j}=\int_{\Omega_{c}} \operatorname{curl} \mathbf{h}_{\gamma}^{\prime} \cdot \mathbf{e}+\int_{\Omega} \mathbf{h}_{\gamma}^{\prime} \cdot \partial_{t} \mathbf{b}=V_{i}-V_{j}
$$

Notice that (74) does not contradict the fact that, in the case of time varying fields, the circulation of e depends on the particular path $\gamma$. 
It has to be remarked that not all couples $\left(\mathbf{a}_{1}, v_{1}\right)$ satisfy the hypothesis of Theorem 1. A notable example of gauge where the hypothesis is not satisfied is the so-called temporal gauge [27] (also called Weyl gauge):

$$
\mathbf{e}_{1}=-\partial_{t} \mathbf{a}_{1}^{*} ; v_{1} \equiv 0
$$

This potential $\mathbf{a}_{1}^{*}$ is sometimes called modified vector potential [32]. Luckily, this is not a problem because, in order to demonstrate the well-posedness of the definition of voltage, it is enough that a single couple of potentials which satisfies the hypothesis of the theorem exists; the uniqueness of voltages is then demonstrated. Moreover, potentials are only intermediate actors in the demonstration and, in practice, it is not necessary to compute them; it is enough that at least a couple of potentials exists. Notice also that, in the case of unbounded domains, Helmholtz decomposition ensures, by itself, the uniqueness of the scalar potential [22], and thus of voltages.

Finally, notice that Theorem 1 requires that the electric field is normal to the ports which connect the computational domain to electric circuits. This is a limitative hypothesis which is required to define voltages but not for currents. Perhaps it is possible to weaken the hypothesis of the theorem so as to define voltages between arbitrary ports (that is, where the electric field is not necessarily normal) based on the power balance. Moreover, it can be conjectured that, in the case of physical electric fields, it is always possible to find at least a couple of potentials $\left(\mathbf{a}_{1}, v_{1}\right)$ which satisfy the hypothesis of Theorem 1 .

The findings on existing Finite Element formulations are resumed in Table 2 (notice that notations may change with respect to the original works). It is found that all of the coupling formulas found in the reviewed scientific literature are particular cases of usage of the newly defined operators $\mathcal{U}_{i j}[\mathbf{e}]$ and $\mathcal{I}_{n}[\mathbf{h}]$.

Table 2. Global quantities expressed by using (34) or (38) in various formulations.

\begin{tabular}{lccl}
\hline Formulation & Reference & Global Quantity & Test Field \\
\hline Electrokinetic & {$[24]$} & $I_{n}$ & $\mathbf{e}^{\prime}=-\operatorname{grad} f_{n}$ \\
Eddy current $\left(\mathbf{t}-\mathbf{t}_{0}-\phi\right)$ & {$[13,23]$} & $U_{n}$ & $\mathbf{h}^{\prime}=\mathbf{t}_{0, n}$ \\
Eddy current $(\mathbf{a}-v)$ & {$[25]$} & $I_{n}$ & $\mathbf{e}^{\prime}=-\operatorname{grad} v_{0, n}$ \\
Eddy current $(\mathbf{h}-\phi)$ & {$[17,26]$} & $U_{n}$ & $\mathbf{h}^{\prime}=\mathbf{c}_{n}$ \\
Stranded coils & {$[7,12,26]$} & $U_{n}$ & $\mathbf{h}^{\prime}=\mathbf{t}_{0}$ or $\mathbf{h}_{s}$ \\
\hline
\end{tabular}

\section{Conclusions}

In this work, the existing scientific literature on methods for coupling external electric circuits with the Finite Element method is critically analysed. It is observed that, in the cases of time varying fields, the definition of "voltage" is at best unclear and usually tainted with unspoken and/or unjustified assumptions.

In order to overcome these problems, a couple of original operators are defined so as to provide workable expressions for voltages and currents. In particular, it has been possible to define voltages based uniquely on the electric field-that is, without relying on any potential. By doing this, a clear physical significance is given to voltages, based on the fact that power balance must be respected. This is a first contribution of this work.

This result restores the "symmetry" between current and voltage, in that current is defined based on the current density only (or, equivalently, on the magnetic field). However, this symmetry is not complete; currents can be uniquely defined through any surfaces (also known as ports), whereas in the case of voltages, the choice of surfaces is constrained by the fact that the electric field must be perpendicular. In the case of static fields, this constraint leads to the notion of equipotential surface (which is flawed for time varying fields); if it were possible to remove this constraint, it would be possible to define a "voltage" between two non-equipotential surfaces! Notice that this is not a problem from the practical point of view because the details of connections between external circuits and computational domains are never modelled accurately in the quasi-static regime. 
By using the newly defined operators, it is possible to devise a general method to write down coupling equations between external circuits and computational domains. In particular, it is demonstrated that all the coupling expressions found in the reviewed scientific literature for the Finite Element method can be retrieved as particular cases of these operators.

It is important to observe that, even if the analysis of existing formulations has been taken on with the Finite Element method only, these new operators do not rely on any particular numerical method. Hence, in principle, they can be used to devise the implementation of coupling between external electrical circuits and computational domains with any other numerical method (cell method, finite difference method, or whatever).

The major limitation of this work is that it is limited to the quasi-static case; in particular, displacement currents are completely neglected. It would be interesting to extend this work in order to take into account capacitive effects $[33,34]$ or even wave propagation.

Funding: This research received no external funding.

Acknowledgments: The author would like to thank A. Bossavit, L. Krähenbühl, G. Meunier and Ruth Sabariego for useful discussions.

Conflicts of Interest: The authors declare no conflict of interest.

\section{References}

1. Tsukerman, I.; Konrad, A.; Meunier, G.; Sabonnadiere, J. Coupled Field-Circuit Problems: Trends and Accomplishments. IEEE Trans. Magn. 1993, 29, 1701-1704. [CrossRef]

2. Leonard, P.; Rodger, D. Voltage Forced Coils for 3D Finite-Element Electromagnetic Models. IEEE Trans. Magn. 1988, $24,2579-2581$. [CrossRef]

3. Leonard, P.; Rodger, D. Modelling Voltage Forced Coils Using the Reduced Scalar Potential Method. IEEE Trans. Magn. 1992, 28, 1615-1618. [CrossRef]

4. Nicolet, A.; Delince, F.; Bamps, N.; Genon, A.; Legros, W. A Coupling between Electric Circuits and 2D Magnetic Field Modeling. IEEE Trans. Magn. 1993, 29, 1697-1700. [CrossRef]

5. Lombard, P.; Meunier, G. Couplage des équations électriques et magnétiques. J. Phys. III 1993, 3, 397-412. [CrossRef]

6. Meunier, G.; Luong, H.; Marechal, Y. Computation of Coupled Problem of 3D Eddy Current and Electrical Circuit by Using T0-T- $\varphi$ Formulation. IEEE Trans. Magn. 1998, 34, 3074-3077. [CrossRef]

7. Bouissou, S.; Piriou, F.; Kieny, C.; Tanneau, G. Numerical Simulation of a Power Transformer using 3D Finite Element Method Coupled to Circuit Equation. IEEE Trans. Magn. 1994, 5, 3224-3227. [CrossRef]

8. Xiang, C.; Xiulian, W.; Yan, L.; Shenghui, W.; Renyuan, T. Transient Simulation of Power Transformers Using 3D Finite Element Model Coupled to Electric Circuit Equations. IEEE Trans. Magn. 2000, 36, 1417-1420. [CrossRef]

9. Piriou, F.; Razek, A. Finite Element Analysis in Electromagnetic Systems Accounting for Electric Circuits. IEEE Trans. Magn. 1993, 29, 1669-1675. [CrossRef]

10. Dreher, T. Couplage de La Méthode Des Éléments Finis Tridimensionnels Avec Une Méthode d'analyse Du Circuit Électrique: Application à La Modélisation Des Machines Électriques Tournantes. Ph.D. Thesis, Institut National Polytechnique de Grenoble (INPG), Grénoble, France, 1994.

11. Gersem, H.D.; Mertens, R.; Lahaye, D.; Vandewalle, S.; Hameyer, K. Solution Strategies for Transient, Field-Circuit Coupled Systems. IEEE Trans. Magn. 2000, 36, 1531-1534. [CrossRef]

12. Dreher, T.; Meunier, G. 3D Modeling of Electromagnets Fed by Alternating Voltage Sources. IEEE Trans. Magn. 1993, $29,1341-1344$. [CrossRef]

13. Meunier, G.; Le Floch, Y.; Guerin, C. A Nonlinear Circuit Coupled t-T0- $\varphi$ Formulation for Solid Conductors. IEEE Trans. Magn. 2003, 39, 1729-1732. [CrossRef]

14. De Gersem, H.; Hameyer, K.; Weiland, T. Field-Circuit Coupled Models in Electromagnetic Simulation. J. Comput. Appl. Math. 2004, 168, 125-133. [CrossRef]

15. Kuo-Peng, P.; Sadowski, N.; Bastos, J.P.A.; Carlson, R.; Batistela, N.J. A General Method for Coupling Static Converters with Electromagnetic Structures. IEEE Trans. Magn. 2007, 33, 2004-2009. [CrossRef]

16. Dular, P.; Henrotte, F.; Robert, F.; Genon, A.; Legros, W. A Generalized Source Magnetic Field Calculation Method for Inductors of any Shape. IEEE Trans. Magn. 1997, 33, 1398-1401. [CrossRef]

17. Dular, P.; Geuzaine, C.; Legros, W. A Natural Method for Coupling Magnetodynamic H-Formulations and Circuit Equations. IEEE Trans. Magn. 1999, 35, 1626-1629. [CrossRef]

18. Dular, P.; Gyselinck, J.; Henrotte, F.; Legros, W.; Melkebeek, J. Complementary Finite Element Magnetodynamic Formulations with Enforced Magnetic Fluxes. COMPEL-Int. J. Comput. Math. Electr. Electron. Eng. 1999, 18, 656-667. [CrossRef] 
19. Dular, P.; Gyselinck, J.; Henneron, T.; Piriou, F. Dual Finite Element Formulations for Lumped Reluctances Coupling. IEEE Trans. Magn. 2005, 41, 1396-1399. [CrossRef]

20. Bossavit, A. What Do Voltmeters Measure? COMPEL-Int. J. Comput. Math. Electr. Electron. Eng. 2008, 27, 9-16. [CrossRef]

21. Van Bladel, J. On Helmholt's Theorem in Finite Regions; Technical Report 440; Midwestern Universities Research Association: Madison, WI, USA, 1958. Available online: http://cds.cern.ch/record/1043670/files/CM-P00066539.pdf (accessed on 23 November 2021).

22. Kettunen, L.; Forsman, K.; Bossavit, A. Formulation of the Eddy Current Problem in Multiply Connected Regions in Terms of $h$. Int. J. Numer. Methods Eng. 1998, 41, 935-954. [CrossRef]

23. Meunier, G. (Ed.) The Finite Element Method for Electromagnetic Modeling; Wiley: London, UK; Hoboken, NJ, USA, 2008.

24. Dular, P.; Legros, W. Coupling of Local and Global Quantities in Various Finite Element Formulations and Its Application to Electrostatics, Magnetostatics and Magnetodynamics. IEEE Trans. Magn. 1998, 34, 3078-3081. [CrossRef]

25. Dular, P.; Henrotte, F.; Legros, W. A General and Natural Method to Define Circuit Relations Associated with Magnetic Vector Potential Formulations. IEEE Trans. Magn. 1999, 35, 1630-1633. [CrossRef]

26. Dular, P.; Kuo-Peng, P.; Geuzaine, C.; Sadowski, N.; Bastos, J.P.A. Dual Magnetodynamic Formulations and Their Source Fields Associated with Massive and Stranded Inductors. IEEE Trans. Magn. 2000, 36, 1293-1299. [CrossRef]

27. Hiptmair, R.; Sterz, O. Current and Voltage Excitations for the Eddy Current Model. Int. J. Numer. Model. Electron. Netw. Devices Fields 2005, 18, 1-21. [CrossRef]

28. Hecht, F. New Development in FreeFem++. J. Numer. Math. 2012, 20, 251-265. [CrossRef]

29. Le Floch, Y. Développement de Formulations 3D Éléments Finis Pour La Prise En Compte de Conducteurs Massifs et Bobinés Avec Un Couplage Circuit. Ph.D. Thesis, Institut National Polytechnique de Grenoble-INPG, Grénoble, France, 2002.

30. Le Menach, Y. Contribution à La Modélisation Numérique Tridimensionnelle Des Systèmes Électrotechniques: Prise En Compte Des Inducteurs. Ph.D. Thesis, Université de Lille, Lille, France, 1999.

31. Dular, P. Modélisation du champ magnétique et des courants induits dans des systèmes tridimensionnels non linéaires. Ph.D. Thesis, Université de Liège, Liège, Belgium, 1996.

32. Emson, C.; Simkin, J. An Optimal Method for 3-D Eddy Currents. IEEE Trans. Magn. 1983, 19, 2450-2452. [CrossRef]

33. Hiptmair, R.; Krämer, F.; Ostrowski, J. A Robust Maxwell Formulation for All Frequencies. IEEE Trans. Magn. 2008, 44, 682-685. [CrossRef]

34. Zhao, Y.; Tang, Z. A Novel Gauged Potential Formulation for 3-D Electromagnetic Field Analysis Including Both Inductive and Capacitive Effects. IEEE Trans. Magn. 2019, 55, 1-5. [CrossRef] 